\title{
INVESTIGACIONES
}

\section{Aprendizaje y producción mediática digital en la escuela: Un abordaje etnográfico del aprendizaje como práctica cultural en Artes Visuales*}

\author{
Learning and digital media production at school: \\ An ethnographic approach to cultural practices in visual Arts Learning \\ Aprendizagem e produção de mídia digital na escola: \\ Uma abordagem etnográfica da aprendizagem como prática cultural em Artes Visuais
}

\author{
Andrea Valdivia Barrios, ${ }^{a}$ Minka Herrera, ${ }^{b}$ Manuela Guerrero ${ }^{c}$ \\ aUniversidad de Chile, Instituto de la Comunicación e Imagen, Santiago, Chile. \\ Teléfono: (56) 2 29787987. Correo electrónico: andrea.valdivia@u.uchile.cl \\ bUniversidad de Chile, Facultad de Ciencias Sociales, Santiago, Chile. \\ Correo electrónico: minkaherrera@gmail.com \\ 'Universidad de Chile, Facultad de Ciencias Sociales, Santiago, Chile. \\ Correo electrónico: maneplu@gmail.com
}

\begin{abstract}
RESUMEN
Las prácticas culturales en el actual escenario de convergencia tecnológica y digital suponen conocimientos y aprendizajes complejos que desafían a la escuela. Este artículo propone un abordaje teórico y metodológico para analizar experiencias educativas que posicionan a los estudiantes como productores mediáticos. Desde el estudio del aprendizaje situado y las prácticas culturales en una etnografía escolar, y tomando un caso de creación artística, se da cuenta de los elementos centrales: actividad, contexto, relaciones, participación y comprensión del proceso y los productos. Los resultados muestran los puntos de inflexión en la práctica pedagógica, las estrategias desplegadas por docente y estudiantes y cómo éstas se articulan con los significados de lo que es aprender en esta situación. Se pretende aportar en el plano educativo con propuestas que acerquen las prácticas mediáticas cotidianas a la escuela, y en la investigación educativa con formas pertinentes de conocer y comprender el aprendizaje.
\end{abstract}

Palabras clave: aprendizaje situado, producción mediática digital, práctica cultural, etnografía, artes visuales.

ABSTRACT
In the current scenario of technological and digital convergence, cultural practices imply both knowledge and learning which defy school as institution. This article proposes a theoretical and methodological approach to analyze educational experiences where students act as media producers. Through an ethnographical research on situated learning and cultural practices at school, applied specifically to a case study of artistic creation, the main situational elements are reported: activity, context, relations, participation and understanding of both process and creations. The results show the turning points in the teaching practice, the strategies used by teachers and students, and how they can be articulated with the common meanings of what learning is in this context. The idea is to contribute to education itself, by putting daily media practices closer to the school context, and also to the research on education, suggesting relevant ways to know and understand learning.

Key words: situated learning, digital media production, cultural practice, ethnography, visual arts.

\footnotetext{
Este trabajo es producto del proyecto Fondeyt No 11130640. Se agradece a Conicyt el apoyo prestado a esta investigación.
} 


\section{RESUMO}

As práticas culturais no atual contexto de convergência tecnológica e digital implicam conhecimento e aprendizagens complexas que desafiam a escola. Propõe-se uma abordagem teórica e metodológica de experiências educativas que posicionam os estudantes como produtores de mídia digital. A partir de um estudo de aprendizagem situado e das práticas culturais em uma etnografia escolar e tomando um caso de criação artística, dá-se conta dos elementos centrais: atividade, contexto, relações, participação e compreensão do processo e dos produtos. Resultados mostram pontos de inflexão na prática pedagógica, as estratégias utilizadas por professores e estudantes e como estas se articulam com os significados do que é aprendido em esta situação. Pretende-se contribuir, no âmbito educativo, com propostas que aproximem as práticas cotidianas de mídia à escola e à pesquisa educativa, com formas pertinentes de conhecer e compreender a aprendizagem.

Palavras chave: aprendizagem situada, produção de mídia digital, prática cultural, etnografía, Artes visuais.

\section{INTRODUCCIÓN}

Las actuales transformaciones en las formas de conocer y en las prácticas comunicativas se han visto intensificadas a partir del desarrollo y convergencia de las tecnologías digitales. Esto plantea importantes desafíos a la educación. Los niños, niñas y adolescentes que hoy habitan las escuelas nacieron en un entorno altamente mediatizado donde la pantalla es quizá el artefacto que más se reitera en su cotidiano (Morduchowicz, 2008). Dichas transformaciones tienen de fondo aprendizajes a nivel técnico, social, semiótico, estético y ético (Buckingham, 2007; Gee \& Hayes, 2011). Abordar estos aprendizajes requiere problematizar aquello que hace relevante la experiencia tecnológica y mediática: las prácticas culturales asociadas.

\subsection{LA RELACIÓN CON LOS MEDIOS Y LAS TECNOLOGÍAS COMO PRÁCTICA CULTURAL}

Las prácticas han sido definidas como formas complejas y reconocibles de actividad social a través de las cuales las personas actúan y significan, manteniendo o transformando, el mundo, a los otros y a sí mismos (Hobart, 2010). Dan cuenta del hacer, pero también de la intención y del sentido de dicho hacer.

Las acciones cotidianas, orientaciones y sentidos en relación con y desde los medios de comunicación en su conjunto dan cuenta de las prácticas mediáticas (Couldry, 2004). Lo mediático indica la particularidad que adopta la comunicación como mediación y construcción permanente de sentidos a partir de la participación de los medios de comunicación de masas (Martín-Barbero, 1987). Esta particularidad es la capacidad de amplificar los sentidos generados y puestos en circulación, sin embargo, tal como señala Jesús Martín-Barbero, la clave está en la mediación como práctica cultural y no en los medios o tecnologías. La digitalización de las tecnologías para la comunicación ha implicado no solo simplificación y aumento en las posibilidades de producir y generar información, de su almacenamiento y circulación, sino que sobre todo, transformaciones en las prácticas culturales asociadas. Según Jenkins, Ford y Green (2013) este cambio en el modelo cultural mediático implica que el "público" deja de tener una posición de recepción activa de mensajes y contenidos y pasa a ser, potencialmente, un productor que da forma y contenido.

Es importante entender esto como un horizonte posible y no como una realidad dada y homogénea de las experiencias juveniles e infantiles, idea que se ha instalado a partir del término Nativo Digital (Prensky, 2001). Diversos estudios han puesto en cuestión esta 
generalización (Kennedy et al., 2010; Sánchez et al., 2010). En el contexto educativo, en particular, se discute la idea de los nuevos aprendices que se asocia con los nativos digitales, Selwyn (2009) plantea que no existe suficiente evidencia empírica y que se requiere de investigación en contexto que incluya las percepciones de los jóvenes.

Cabe preguntarse cómo la escuela atiende a estos desafíos, cómo profesores, profesoras y estudiantes experimentan en aprendizajes relacionados con prácticas de producción mediáticas digitales; y cómo estudiar y conocer estos procesos de aprendizajes. En este artículo pretendemos aportar en ambos sentidos desde una investigación que está en curso, a través de una discusión teórico conceptual del aprendizaje y la producción mediática como prácticas situadas, su abordaje por medio de la etnografía y la ejemplificación en un caso concreto en artes visuales.

\subsection{PRODUCCIÓN MEDIÁTICA DIGITAL Y APRENDIZAJE EN LA ESCUELA CHILENA}

Desde el campo de la comunicación-educación la producción y creación mediática digital de los y las estudiantes tiene especial valoración, dado que los posiciona en un rol activo y les ofrece experiencias complejas de aprendizaje (Buckingham, 2007; Sadik, 2008). Supone avanzar desde una lógica que entiende a los medios y las tecnologías como recursos didácticos al servicio de procesos pedagógicos instruccionales, hacia una donde se atienden las prácticas mediáticas en escenarios culturales concretos, y a partir de allí se promueven aprendizajes.

La situación en Chile a nivel de política educativa presenta ciertas paradojas en este tema. A pesar de la sistemática inversión en el Programa de Informática Educativa, cuyo énfasis ha estado en equipamiento, acceso y formación docente (Instituto de Informática Educativa, Universidad de La Frontera, 2010), la evaluación desde el 2011 evidencia que el desarrollo de habilidades tecnológicas complejas en estudiantes no logra resultados satisfactorios y que se mantienen las brechas de desigualdad social (Centro de Educación y Tecnología, CEPPE y Fundación País Digital, 2013; Centro de Educación y Tecnología, 2014). Ruiz (2013) en una investigación en establecimientos de la Región Metropolitana muestra cómo las prácticas tecnológicas de los adolescentes dan cuenta de un nivel de uso más bien básico, mientras que hacen una marcada distinción entre las prácticas cotidianas asociadas a la comunicación y entretención, y las prácticas tecnológicas escolares asociadas al deber y un aprendizaje escolar instruccional.

En el plano curricular el escenario es confuso. Los Objetivos Fundamentales Transversales (OFT) del Marco Curricular (Ministerio de Educación, 2009) indican como uno de sus ejes las Tecnologías de Información y Comunicación, las que aparecen promoviendo el desarrollo de diversas habilidades para "manejar el mundo digital, para desenvolverse en él en forma competente y desarrollar en alumnas y alumnos una actitud reflexiva y crítica, que les permita comprender y participar activamente como ciudadanos" (p. 23). Cuando se entra al plano de la concreción curricular en un primer nivel, el de los Planes y Programas de las asignaturas o sectores de aprendizaje, no existe posibilidad que las habilidades propuestas en los OFT sean desarrolladas. Los dos subsectores que guardan relación con la producción mediática digital son Lenguaje y Comunicación y Artes Visuales. En el primero se observa un abordaje limitado con una orientación de los medios de comunicación como recursos didácticos complementarios (De Fontcuberta, 2009). En las Artes Visuales se aprecia una orientación que releva principalmente la dimensión expresiva de la plástica y el conocimiento teórico (Gíraldez y Pimentel, 2011). 
Estudios Pedagógicos XLI, Nº Especial: 231-251, 2015

APRENDIZAJE Y PRODUCCIÓN MEDIÁTICA DIGITAL EN LA ESCUELA: UN ABORDAJE ETNOGRÁFICO DEL APRENDIZAJE COMO PRÁCTICA CULTURAL EN ARTES VISUALES

En este contexto, es un desafío que la escuela promueva de manera sistemática aprendizajes complejos en relación con la producción mediática digital. La investigación sobre prácticas pedagógicas e inclusión de las TIC muestra bajos logros, se mantienen formas tradicionales de entender y desarrollar la enseñanza y el aprendizaje (Arancibia y Badia, 2013; Instituto de Informática Educativa Universidad de La Frontera y ADIMARK Gfk, 2013). Las pocas experiencias encontradas están relacionadas con iniciativas innovadoras asociadas a espacios extracurriculares dentro de la escuela tales como radio o televisión escolar digital (Cocq, 2015), o promovidas por proyectos universitarios, como el caso de Kelluwen en el sur de Chile, que busca desarrollar experiencias pedagógicas que incorporen la creación de contenidos digitales (Scheihing et al., 2013).

\section{APRENDIZAJE Y PRODUCCIÓN MEDIÁTICA DIGITAL COMO PRÁCTICAS CULTURALES SITUADAS}

Las prácticas han sido definidas como formas complejas y reconocibles de actividad social y articulación, a través de las cuales las personas mantienen o cambian a sí mismas, a otros y al mundo, y sobre las cuales varían sus condiciones. Supone la articulación de sujetos, objetos y propósitos. No son lineales ni consistentes, pueden exhibir contradicciones. Esto se puede entender si se asume una mirada antropológica de las prácticas, sin buscar una definición universal de ellas. Según Hobart (2010) la práctica siempre es situada. El autor plantea que las prácticas no son solo culturales o históricas, sino también situadas y contingentes. La práctica es un marco de referencia que usamos para interrogar una compleja realidad, por lo tanto depende de que sea identificada como tal y de que un sujeto articule una serie de actividades o eventos como práctica.

Abordar los aprendizajes que desarrollan las y los estudiantes producto de su participación en actividades y experiencias de producción de medios requiere de una concepción del aprendizaje que reconozca las particularidades de los escenarios, contextos, actividades y mediadores en juego en dichas experiencias. Ello implica entender el aprendizaje como un fenómeno social, histórico y situado (Lave, 1996; Vigotsky, 1978; Wertsch, 1991). No solo se aprende sobre un contenido sino que también se aprende a participar en el escenario, adentrándose en las prácticas y las normas que organizan la participación en una comunidad de práctica (Lave \& Wenger, 1991; Gee, 2004).

\subsection{ACTIVIDAD, CONTEXTO Y RELACIONES. CLAVES PARA ENTENDER EL APRENDIZAJE COMO PRÁCTICA}

Desde la perspectiva que asumimos, el aprendizaje está siempre presente independiente de que exista la intención explícita de su promoción, o un escenario institucionalizado para dichos fines. Esto porque la complejidad de la vida social requiere de cambios constantes en el conocimiento y la acción de nuestra adaptación y comprensión. Las teorías de la actividad situada no separan entre acción, pensamiento, sentimiento y valor; tampoco entre la dimensión colectiva e histórico-cultural de la actividad y su forma localizada, siempre significativa, interesada y conflictiva. Para entender lo particular del enfoque es necesario desarrollar qué se entiende por aprendizaje y conocimiento. 


\subsubsection{Participación y comprensión en el aprendizaje situado}

El aprendizaje como práctica da cuenta siempre del escenario y actividad en que ocurre. La mente en acción, en palabras de Wertsch (1991), no es otra cosa que la forma en que se entiende aprendizaje y desarrollo en esta concepción; más allá de la adquisición y de la idea de la mente como caja negra donde ocurre la cognición. Existe una gran distancia entre esta forma de entender el aprendizaje y las teorías cognitivistas clásicas. Lo que ha llevado, según Lave (1996), a varios investigadores socioculturales a alejarse del término Aprendizaje, evitando el sentido tradicional, abordando el fenómeno a través de los conceptos de Participación y Comprensión. La Participación da cuenta de la acción e involucramiento de los sujetos en las actividades sociales, es decir, lo que hacen, su intencionalidad y su motivación. La Comprensión se refiere al sentido y forma de entender que tienen los participantes: actividad (tarea, propósito, producto, herramientas, etc.), participación y aprendizaje. Es un proceso parcial y abierto y, a pesar de ello, no es azaroso ni infinito, gracias a los encuadres y orientaciones que da la propia actividad.

Participación y comprensión contribuyen a entender el aprendizaje como un proceso que transforma a sus involucrados, a visualizar la forma en que se genera nuevo conocimiento y cómo este se pone al servicio de la participación en el escenario.

\subsubsection{Conocimiento como proceso y producto cambiante}

Desde esta perspectiva, el conocimiento está en estado de cambio, se define por su valor de uso en la actividad social y desde allí la idea de construcción de conocimiento es relevante. El conocimiento no se absorbe, pues si bien la relación con él puede tener una dimensión de adquisición de información, lo relevante es la acción de reconceptualización que deben hacer los sujetos para usarlo y ponerlo al servicio de la participación en la actividad. Así, el conocimiento siempre tendrá algo nuevo. Lave (1996) plantea que el sentido cambiante del conocimiento también dice relación con el carácter situado del enfoque, la participación de diversos sujetos implica a su vez heterogeneidad en la posición social, intencionalidad y posibilidades subjetivas.

Para conocer y comprender el aprendizaje y la producción mediática digital en tanto práctica cultural es necesario atender a dos elementos centrales e interrelacionados que la constituyen: Actividad y Contexto.

La Actividad se asocia con límites temáticos, normas procedimentales, propósitos que ordenan la participación en ella, herramientas mediadoras (simbólicas y materiales) y productos. El Contexto es entendido como un mundo social constituido en la relación de los sujetos en acción. Contexto no como el espacio estructural en que ocurre la actividad, sino como las relaciones inmediatas e históricas que están presentes y permiten el curso de ésta. La dimensión histórica es la que da estabilidad y sentido a la actividad, pues toda acción particular se constituye socialmente y adquiere su significado a partir de la ubicación que tiene en sistemas de actividad generados social e históricamente.

Tanto la producción mediática digital, manifestación de la práctica mediática, como la práctica pedagógica pueden ser entendidas como sistemas de actividades que suponen determinados contextos históricos y relacionales, y particulares significados, conocimientos y pautas normativas que orientan y se configuran desde la comprensión de los participantes. 
Estudios Pedagógicos XLI, N Especial: 231-251, 2015

APRENDIZAJE Y PRODUCCIÓN MEDIÁTICA DIGITAL EN LA ESCUELA: UN ABORDAJE ETNOGRÁFICO DEL

APRENDIZAJE COMO PRÁCTICA CULTURAL EN ARTES VISUALES

No toda acción que es parte de una actividad es objeto de análisis, solo aquellas que otorgan sentido y se vinculan con relaciones más allá de la situación. El análisis debe identificar con claridad acciones, operaciones, significados y sistemas de actividad. El significado se puede enlazar con el proceso de comprensión dentro de la práctica. Säljö y Wyndham (1996) sostienen que el significado de la tarea no puede ser definido independiente de la actividad ni de los supuestos que los sujetos tienen sobre las premisas relevantes de la acción.

Con esta articulación de elementos cabe preguntarse cómo estudiar el aprendizaje y la producción mediática digital como práctica; lo que abordaremos a continuación.

\subsection{EL ESTUDIO DEL APRENDIZAJE Y LA PRODUCCIÓN MEDIÁTICA COMO PRÁCTICA. EL APORTE DE LA ETNOGRAFÍA}

Si asumimos que la práctica es un fenómeno complejo y contradictorio, su estudio requiere de un abordaje igualmente complejo. Según Hobart (2010) su característica situada y de orientación normativa hace necesario que la investigación de la práctica considere la observación de la actividad como proceso y resultado, y la atención a los significados que los actores atribuyen tanto a su propia práctica como a la lectura que haga el o la investigadora de ésta. Desde nuestra perspectiva, la etnografía, como enfoque y método, resulta pertinente.

La etnografía como enfoque da cuenta de una concepción y una práctica de conocimiento que tiene como propósito comprender los fenómenos culturales y sociales desde la perspectiva de los miembros del grupo o comunidad respecto de la cual se genera conocimiento (Guber, 2011). Lo que hace particular a esta aproximación es el foco en la vida cotidiana de las personas. Esto implica interrogar y describir interpretativamente lo que hace la gente, los significados que dan a sus experiencias y los contextos políticos, sociales, materiales y económicos (Anderson Levitt, 2006). El esfuerzo está en abordar la complejidad de la vida social con una mirada holística.

Este abordaje requiere de una doble perspectiva en el trabajo etnográfico: una comprensión del punto de vista del grupo o comunidad observado para acercarse a la lógica de sus acciones, y una toma de distancia para "ver" aquello que resulta obvio o invisible por la naturalización con que operan las orientaciones. Esta doble perspectiva da cuenta de la reflexividad tan característica del trabajo etnográfico. Según Guber (2004) esta reflexividad no solo opera en el momento del análisis alejado del "campo", sino que debe ser permanente. Cuando se está dentro del campo la reflexividad supone la interacción entre las reflexividades del o la observadora y la de los actores sociales.

La etnografía como método supone un continuo de decisiones en el terreno que exigen rigurosidad y flexibilidad de quien investiga, tanto en su estar y transitar en el espacio social observado, en el encuadre de sus observaciones y conversaciones, en la producción de la información y en el análisis sistemático. Todas estas actividades ocurren la mayoría de las veces de manera simultánea y en interacción. Las preguntas se revisan constantemente y esta revisión siempre considera la tensión con los mismos actores. Se entra y sale del "campo" una y otra vez. De ahí que se valore el tiempo de permanencia del etnógrafo o etnógrafa en el escenario estudiado. ¿Cómo observar y entender el aprendizaje en determinadas actividades?, ¿cómo dar cuenta del proceso de producción y los diversos contextos relacionales, inmediatos y mediatos e históricos, si no es participando en ese proceso?

En las siguientes secciones daremos cuenta de cómo estamos abordando metodológicamente la etnografía durante el primer año, 2014, y cómo hemos ido construyendo el 
análisis de los aprendizajes y la producción mediática digital en dos escuelas de Santiago de Chile, una pública y una privada. Hemos tomado uno de los casos y dentro de él una unidad de aprendizaje en Artes Visuales para ejemplificar y compartir hallazgos relevantes.

\section{ESTUDIO DE CASO Y MÉTODO}

\subsection{EL CASO DE ESTUDIO}

La escuela seleccionada es un establecimiento privado de la Región Metropolitana, que cuenta con una propuesta curricular donde la producción mediática digital está presente en una amplia oferta electiva y dentro de algunas actividades específicas en las asignaturas de Lenguaje y Comunicación y Artes Visuales. Este fue el principal criterio para la búsqueda de las escuelas, otros fueron la representación de dependencia diferenciada, pública y privada, y la voluntad de participar de la dirección y de los docentes.

Ubicado en una comuna del sector nororiente de la capital, compuesta por grupos socioeconómicos medio-altos y altos, y que según información del municipio tiene la mayor concentración de profesionales con estudios universitarios del país, el colegio tiene una matrícula total de 1.672 estudiantes, un promedio de cuatro cursos por nivel y 28 estudiantes por curso ${ }^{1}$, con oferta completa para el sistema pre-escolar y escolar.

\subsection{UNIDADES Y PROCESO DE OBSERVACIÓN}

El ingreso al campo ocurrió en mayo del 2014, luego de una serie de reuniones con la dirección. Nuestra primera actividad, junto con el inicio de la observación, fue una reunión con docentes seleccionados por la dirección a partir del criterio que le plateamos: profesores de asignatura o taller electivo que desarrollan actividades de producción mediática digital con sus estudiantes. El primer año el foco del estudio estuvo puesto en las prácticas pedagógicas como marco de los aprendizajes de los y las estudiantes, y en la caracterización de sus prácticas mediáticas. Trabajamos la observación de manera intensiva con 4 profesores y 2 profesoras, en dos cursos: octavo básico y primero medio, y tres talleres electivos. La observación de aula ocurrió de manera semanal durante siete meses.

Las unidades de observación fueron: A) el aula de un octavo básico y un primero medio, durante las clases de Lenguaje y Comunicación y Artes Visuales. B) tres talleres relacionados con la creación audiovisual, el cine y el periodismo escolar. C) la escuela como contexto histórico y relacional a través de: i. documentos históricos e institucionales (libro, documental, informes, etc.) ii. instancias de encuentro y actividades de ocio y recreación dentro del establecimiento (recreo, almuerzo, eventos culturales y sociales planeados por dirección, docentes, estudiantes y ex alumnos). D) grupos y páginas de Facebook, soporte virtual de actividades educativas de los talleres electivos. E) el barrio.

Los resultados que presentamos son parte del estudio de octavo básico en las clases de Artes Visuales, en una de las dos unidades de aprendizaje observadas. 
Estudios Pedagógicos XLI, N Especial: 231-251, 2015

APRENDIZAJE Y PRODUCCIÓN MEDIÁTICA DIGITAL EN LA ESCUELA: UN ABORDAJE ETNOGRÁFICO DEL

APRENDIZAJE COMO PRÁCTICA CULTURAL EN ARTES VISUALES

\subsection{TÉCNICAS Y MATERIALES}

La etnografía ha considerado un conjunto de técnicas para producir información. La observación directa y participante como estrategia central; entrevista semi-estructurada con docentes antes de la observación de aula y al final del primer año, y con estudiantes al final de las unidades de aprendizaje para recoger significados sobre el proceso, aprendizajes y productos; revisión documental para textos escritos, visuales y audiovisuales. También utilizamos un cuestionario para conocer las prácticas y competencias mediáticas de los y las estudiantes.

El volumen de materiales e información producidos es significativo. Reportes e informes escritos de cada unidad de observación, audios y transcripción de las entrevistas, fotografías, videos, recopilación de los productos creados por estudiantes, documentos escritos y audiovisuales producidos por la escuela.

En el caso de la unidad de observación que presentamos: Artes visuales en octavo básico, unidad de aprendizaje Desarrollo de las Artes Visuales en el siglo XX, la profesora desarrolló la actividad: Nanometraje: Ser-Cubo-Contexto. La actividad tuvo una duración de 15 sesiones de 90 minutos, una vez por semana, entre los meses de mayo a agosto. Los materiales producidos y recolectados fueron: 15 registros de observación con un número aproximado de 90 páginas; 75 fotografías y 10 videos de registro; 2 entrevistas a la docente; 6 entrevistas con grupos de estudiantes, con una duración promedio de 45 minutos y 18 participantes en total; 10 nanometrajes como creaciones audiovisuales digitales.

\subsection{ANÁLISIS}

El proceso de análisis consideró en su etapa inicial una aproximación inductiva que consistió en la revisión, organización y clasificación de los materiales (notas de campo, fotografías, transcripción de las entrevistas, producción de los estudiantes, registros de facebook). Guiadas por las preguntas de investigación y a partir de un análisis categorial, buscamos configurar patrones y tendencias. A partir de lo anterior levantamos algunas claves interpretativas organizadas en las siguientes dimensiones de la práctica pedagógica: aprendizaje, evaluación-retroalimentación, relación entre estudiantes y relación docente y estudiantes. En esta etapa volvimos al establecimiento y generamos talleres de análisis conjunto con docentes y estudiantes para incluir sus análisis e interpretaciones respecto de un corpus de materiales seleccionados. Esta última actividad analítica está en procesamiento, por lo que los resultados que presentamos corresponden a la primera etapa, sin embargo, atienden a la remirada que nos dejó el diálogo y discusión con docentes y estudiantes. La calidad del análisis ha sido guiada por la pertinencia y coherencia con las preguntas de investigación iniciales y aquellas generadas durante el trabajo de campo (Bergman \& Coxon, 2005) y por el ejercicio reflexivo del equipo (Guber, 2004).

\section{APRENDIZAJE Y PRODUCCIÓN MEDIÁTICA DIGITAL EN UN CASO EN ARTES VISUALES. ALGUNOS RESULTADOS ETNOGRÁFICOS}

Organizamos esta sección a partir de las dos claves del estudio de las prácticas: Actividad y Contexto, entendiendo que las Relaciones, componente también central en el estudio de las prácticas, es transversal a las dos y en particular define el contexto. 


\subsection{EL LICEO COMO CONTEXTO SITUADO}

\subsubsection{Origen público y de experimentación pedagógica}

De acuerdo a un documental realizado por docentes y estudiantes, el liceo se crea el año 1932 en el marco de un debate educativo nacional en torno a la Ley de Instrucción Primaria. Un grupo de pedagogos "progresistas" cuestiona las formas tradicionales de enseñanza y de entender la educación. Desde este grupo se propone la fundación de un establecimiento con dependencia directa del Ministerio de Educación, cuyo propósito era transformarse en un centro de experimentación curricular y pedagógica, que atendiera tanto a la idiosincrasia nacional como a las nuevas exigencias de un país que marchaba en busca de su propio destino $^{2}$. Además, es el primer establecimiento en impartir coeducación donde niños y niñas se educan juntos.

En el plano pedagógico, la experimentación estaba orientada por la promoción del aprendizaje activo y la necesidad de generar nuevo conocimiento teórico y práctico en pedagogía. Se plantea que los profesores aportan a la formación humana; la comunidad educativa se configura a partir de la horizontalidad entre profesores y alumnos; existe heterogeneidad política y religiosa, junto con una marcada participación política y social de los actores escolares.

El año 1942 el establecimiento es traspasado a la primera universidad pública del país. Durante la Dictadura Militar el establecimiento, al igual que todo el sistema educativo, vive importantes transformaciones que van desde su dependencia administrativa, ahora privada, hasta la reducción de las posibilidades de experimentación pedagógica.

\subsubsection{El liceo actual desde la caracterización social y educacional}

Actualmente el énfasis del proyecto educativo es el desarrollo integral y valórico, y la excelencia académica. Con una orientación laica, tiene por misión crear una comunidad escolar participativa, crítica y pluralista.

El establecimiento se ubica en una comuna urbana del sector oriente de la Región Metropolitana, en un barrio residencial, a metros de una de sus principales avenidas. Próximo a la Municipalidad, y a la principal plaza de la comuna que cuenta con teatro, iglesia, biblioteca, sucursales bancarias, centro de formación educacional y restaurantes.

La mayoría de los y las estudiantes residen en la misma comuna. Los y las apoderados, padres y madres por lo general, son egresados, profesores de la misma escuela o profesores universitarios. Según información del Ministerio de Educación, la mayoría de los apoderados ha declarado tener 16 o más años de escolaridad en el caso del padre y 15 o más en el caso de la madre ${ }^{3}$. El establecimiento posee un $8 \%$ o menos de estudiantes en condición de vulnerabilidad social.

$\mathrm{Al}$ interior de la escuela los y las estudiantes cuentan con una gran extensión de áreas verdes, espacios amplios para realizar actividades físicas tanto en zonas abiertas como cerradas, y un aula magna donde se realizan actividades culturales.

Sitio Web Institucional

http://www.simce.cl/ 
Estudios Pedagógicos XLI, Nº Especial: 231-251, 2015

APRENDIZAJE Y PRODUCCIÓN MEDIÁTICA DIGITAL EN LA ESCUELA: UN ABORDAJE ETNOGRÁFICO DEL APRENDIZAJE COMO PRÁCTICA CULTURAL EN ARTES VISUALES

En cuanto al diseño curricular, la escuela adapta los Programas de Estudio Nacional de acuerdo a los requerimientos de cada sector de aprendizaje. En el caso de Artes Visuales se profundiza en los Objetivos Fundamentales Transversales, enfatizando en la formación ética: "autorregulación de la conducta y autonomía, sentido de trascendencia, vocación por la verdad, la justicia, el espíritu de servicio y el respeto por el otro"4.

Cada sector de aprendizaje asigna un profesor que debe planificar anualmente un nivel. Luego cada profesor tiene la libertad de modificar las actividades tomando en consideración las características de cada curso. Además, cada departamento crea y oferta electivos, tales como: cortometraje, periodismo, serigrafía, telar, etc.

\subsubsection{El $8^{\text {vo }}$ y la clase de Artes Visuales}

El curso está compuesto por 28 estudiantes, 13 hombres y 15 mujeres entre 13 y 14 años de edad, la mayoría juntos desde pre-escolar. Es posible identificar grupos con límites simbólicos distintivos. En el caso de las mujeres, hay un grupo que las une el gusto por el "animé japonés", son adolescentes que tienen una personalidad más bien retraída y participan de comunidades virtuales donde crean contenidos mediáticos. Un segundo grupo corresponde a adolescentes que participan en scouts, talleres de danza, pintura y batucadas, son más extrovertidas y demuestran cierto liderazgo en el curso. Los hombres se diferencian por su participación en el aula, están los que participan de las conversaciones en clases y aquellos más introvertidos.

En general, nos llamó la atención la autorregulación que la mayoría de los y las estudiantes mostraron al participar en diversas situaciones didácticas o convivenciales, así como la dinámica de interacción y participación donde era habitual la expresión de diferentes puntos de vista y la crítica respetuosa sobre el trabajo o quehacer del compañero, sin ser percibido como amenaza o agresión.

Respecto a la clase de Artes Visuales, los y las estudiantes se muestran relajados. Se desplazan constantemente por la sala, escuchan música mientras trabajan, conversan con sus compañeros y ocupan el espacio de manera diversa: se sientan sobre mesas, sillas o también en el suelo (Imagen 1). La relación entre docente y estudiantes, como veremos, es cercana a la vez que asertiva, observamos diversas situaciones donde la profesora los tensiona para que reflexionen, pregunten y puedan expresarse libremente.

\subsection{LA ACTIVIDAD COMO PRÁCTICA PEDAGÓGICA PARA LA CREACIÓN MEDIÁTICA DIGITAL}

La actividad de aprendizaje Nanometraje: Ser-Cubo-Contexto fue especialmente incluida y desarrollada por la docente para participar en el proyecto, era primera vez que la realizaba, lo que implicó una adecuación a la planificación de la asignatura para 8vo básico.

La propuesta pedagógica implicó la creación, en duplas, de un nanometraje a partir del desarrollo paulatino de tres recursos creativos: un ser, un cubo y un contexto. La docente explicaría esto en la entrevista como: forma, volumen, mensaje artístico y contenido visual.

En la planificación docente, los aprendizajes esperados declarados fueron: desarrollar una idea, sentimiento o emoción en una expresión creativa y técnica; identificar características de la expresión tridimensional (cubo); incluir en la experiencia artística -

Objetivos Fundamentales Transversales extraídos del Programa de Estudio de Artes Visuales realizados por el Liceo. 
Imagen 1. Fotografía $8^{\circ}$ en clases de Artes visuales

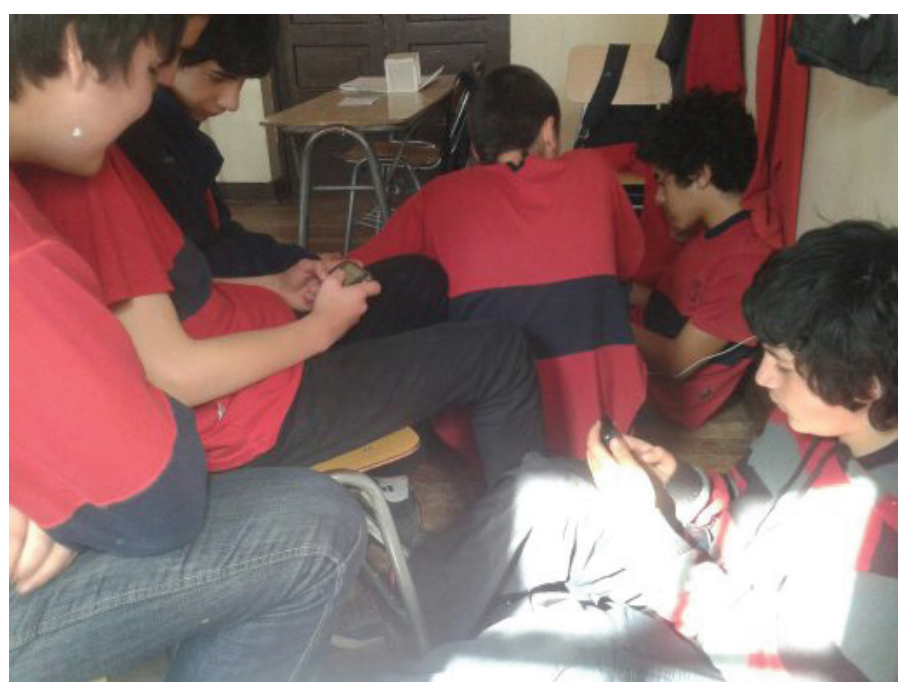

tanto en el plano, la tridimensión y el audiovisual-, ideas y percepciones sobre fenómenos humanos, estéticos y culturales; manifestar juicios críticos sobre las propias creaciones y sus pares y obras. En términos narrativos, la docente esperaba la creación de una historia audiovisual de corta duración que presentara una situación puntual y se resolviera de manera rápida, sorpresiva e inesperada. El desafío principal era transmitir un mensaje.

La planificación se organizó en cuatro fases. I: creación del ser en formato plano. Dibujo con lápices en una hoja de papel con la representación del ser como sí mismo o una alteridad. II: construcción de un cubo. Para ello la profesora mostró un vídeo con indicaciones de cómo construirlo y les facilitó una plantilla de un cubo para que ellos y ellas lo realizaran en un cartón del tamaño que desearan. III: planificación del contexto en el que se situaba la historia. Por último, IV: conjugación de los tres elementos (ser-cubo y contexto) para grabar y montar la historia.

En cada una de las fases, la profesora presentó nanometrajes y películas para nutrir el proceso de creación de los y las estudiantes. Dentro de los materiales solicitados además de los papeles, cartulinas, pegamento y materiales pictóricos o de dibujo, la docente incluyó artefactos de registro audiovisual domésticos (celulares con cámara u otros dispositivos electrónicos), central para el trabajo propuesto.

\subsubsection{El Nanometraje y su particularidad artística-comunicativa}

Los nanometrajes corresponden a la categoría de microrrelato y son parte de la tendencia del Minimalismo en las Artes Visuales del siglo XX. Ha logrado su desarrollo gracias a la circulación en redes sociales. Con un formato de texto audiovisual breve, suelen contemplar solo un concepto e idea durante su filmación. Según Guarinos y Gordillo (2010) los nanometrajes se caracterizan por: una relevancia simbólica y representacional de la imagen, pues en escaso tiempo deben sugerir lo que piensan los personajes y su contexto histórico; 
estructuras narrativas sencillas donde solo existe una secuencia o escena representada; y personajes que representan estados más que actuaciones. Otro aspecto característico es el uso de dispositivos domésticos para su producción.

En el caso de la experiencia analizada, la docente deja todas estas características como elementos a explorar e indagar a partir de la creación, sin abordarlos explícitamente como contenidos que encuadran u orientan las posibilidades creativas de los y las estudiantes, salvo el requisito tecnológico para la grabación y la duración.

\subsubsection{Proceso artístico y creativo del nanometraje: el valor de la experiencia}

La creación del nanometraje fue un proceso extenso. La docente había planeado esta actividad en ocho sesiones organizadas en cuatro fases. No obstante, se extendió y tuvo una duración de 15 sesiones. Se cruzaron dos procesos con lógicas paralelas: una lógica secuencial de la actividad planeada por la profesora, con un comienzo, un desarrollo y un cierre, y que tuvo su manifestación central en el formato de la planificación, la que intentó seguir la docente; y una lógica recursiva y flexible de la creación artística como proceso vivido por todos los participantes (Figura 1).

Figura 1. Procesos de producción del nanometraje

\section{NANOMETRAJE COMO PROCESO SECUENCIAL}

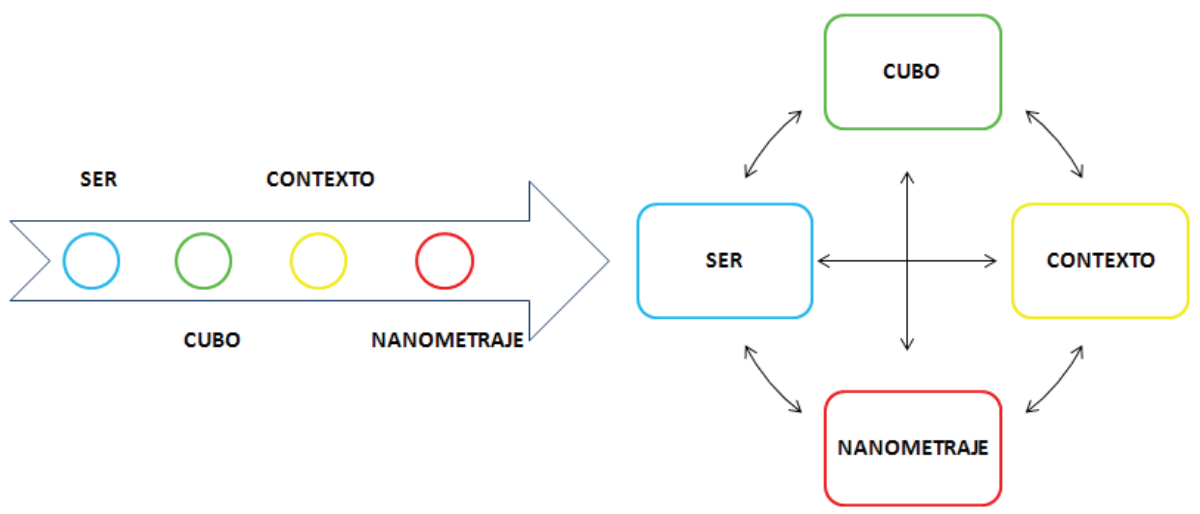

\subsubsection{Nanometraje como proceso recursivo y flexible para la creación}

La clase de Artes Visuales se realizaba los días miércoles entre las 9:45 y las 11:15 de la mañana. Después del segundo recreo sonaba el timbre, los estudiantes se dirigían a su sala y esperaban en su interior a la profesora. La docente siempre corriendo y apurada dejaba el libro de clases, el computador, los parlantes y su mochila sobre el escritorio y de pie se paraba frente a los estudiantes. Estos, de pie, conversaban, reían, cantaban y tocaban guitarra; parecían no prestar atención a su llegada. La profesora entonces comenzaba a ingeniárselas para llamar su atención. Generalmente alzaba la voz, salvo una vez que llegó 
con una matraca de juguete. Con un tono de voz elevado pero muy amable decía: ¿hola monitos, cómo están? Al ver que los estudiantes seguían conversando alzaba aún más la voz y en tono serio y algo molesto decía: ¿me parece exagerado a estas alturas hermano?; ¿hermano no regale su plata? ¡Ponga atención!; un minuto de su atención por favor. Luego de unos minutos, un par de estudiantes comenzaba a hacer callar a sus compañeros y, finalmente, lograba la atención de los estudiantes y podía comenzar la clase.

La clase, en general, se desarrollaba con un inicio donde la profesora recordaba en qué parte de las fases de creación estaban, mostraba distintos nanometrajes y continuaba con tiempo para que los estudiantes realizaran los productos para el nanometraje. Esto dentro de la contingencia que tiene la dinámica de aula donde, hay un sinnúmero de emergentes a los que la docente tendía a atender y conducir hacia su intención pedagógica. La sesión del miércoles 04 de junio es un buen ejemplo de esto:

La profesora convoca nuevamente a los estudiantes a la biblioteca para iniciar la Segunda Fase del proyecto: construcción de un cubo. Ella tenía preparado un video donde se explica paso a paso como hacer un cubo de manera didáctica, sin embargo, los estudiantes tenían como foco otra situación, ver en YOUTUBE “Niño infestado de Piojos, ¡Increíble!”. Los estudiantes sentados en sus sillas y la profesora de pie frente ellos. De fondo, en la pantalla se proyecta el inicio de YOUTUBE. Un estudiante se para y escribe en el computador portátil: Niño infestado de Piojos. Los estudiantes que ya lo habían visto decían: es súper bueno profe. La profesora accede y todos vemos el video. Se presenta una situación en la que participan una mujer y un niño. En un primer plano se ve la nuca del niño, tiene el cabello corto, oscuro y liso, y húmedo. El rostro de la mujer no se muestra en ningún momento. Ella sostiene un peine metálico especial para pediculosis. La mujer, con rapidez, arrastra el peine por el cabello del niño una y otra vez. Luego de un minuto, la mujer acerca el peine a la cámara. Se observa una mancha oscura que cubre la mitad del peine. Por un momento pensé que era pelo, pero estaba equivocada, se trataba de una comunidad de piojos vivos que se movían con rapidez desplazándose en distintas direcciones.

Los estudiantes comenzaron a decir en voz alta, todos al mismo tiempo (con repulsión): ¡Qué asco! ¿acaso no se bañaba? ¡que sucio el niño ¡que asqueroso la textura!

La profesora, de pie, levanta su mano y alza la voz: La imagen activó algo en mí. Texturas con vida, comunidad, se me imagina, asocié, cuerpo geométrico a textura, relieve, contexto, ser.

En ese momento comienzan los y las estudiantes a escuchar a la profesora. Ella, de manera pertinente y repentina, vincula la experiencia audiovisual con la actividad del ser, cubo y contexto, preguntando:

Profesora: En relación al contexto ¿qué piensan?

Un estudiante: Es asqueroso.

Una estudiante: La textura se mueve.

Profesora: ¿Y el ser?

Estudiantes: Está molesto, no se bañaba.

Es interesante observar en esta situación cómo la docente atiende la intención momentánea de los estudiantes, ver el video de los piojos, motivación distinta a la propia. La docente acepta que todos vean el video antes de iniciar la actividad planificada. Lo hace y luego vincula el contenido del video con la actividad de producción mediática. Esto por medio de dos aspectos relevantes: la experiencia personal que provocó el video desde una dimensión emocional, y el análisis del material desde las claves creativas del nanometraje: contexto, ser, cubo, textura, volumen. 
Estudios Pedagógicos XLI, N Especial: 231-251, 2015

APRENDIZAJE Y PRODUCCIÓN MEDIÁTICA DIGITAL EN LA ESCUELA: UN ABORDAJE ETNOGRÁFICO DEL APRENDIZAJE COMO PRÁCTICA CULTURAL EN ARTES VISUALES

Volviendo a un plano general, el cotidiano del proceso creativo ocurrió tanto dentro de la sala como fuera de ella, en las casas de los estudiantes. Nosotras pudimos observar de manera directa la actividad solo en el aula. Los y las estudiantes durante la clase se desplazan por la sala, conversan con sus compañeros, escuchan música escogida por ellos, trabajan en el suelo o simplemente piensan. En una ocasión un estudiante estuvo callado mirando al suelo casi toda la clase, de pronto la profesora se acerca y le dice ¿está pensando hermano? Registre lo que piensa, le puede servir para más adelante.

La dinámica más flexible que permite el desarrollo y manifestación de la individuación para el proceso creativo se expresa en la Imagen 2. En ella observamos a un adolescente con audífonos, sentado con los pies sobre la mesa, concentrado viendo algo que tiene entre las piernas; más lejos, dos estudiantes, uno de pie y otra sentada sobre la mesa parecen revisar algo; y en el extremo inferior derecho de la fotografía, casi saliendo del encuadre, un estudiante con el capuchón de su chaqueta cubriendo la cabeza mira su celular, frente a él sobre el escritorio, un cubo blanco y negro parece terminado. La Imagen 3, nos muestra una estudiante concentrada en la producción del cubo.

Imagen 2. $8^{\circ}$ en clase de Artes Visuales

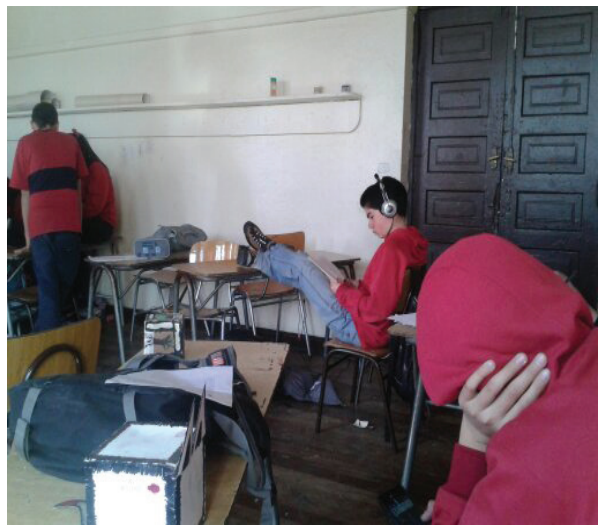

Imagen $3.8^{\circ}$ en construcción del cubo

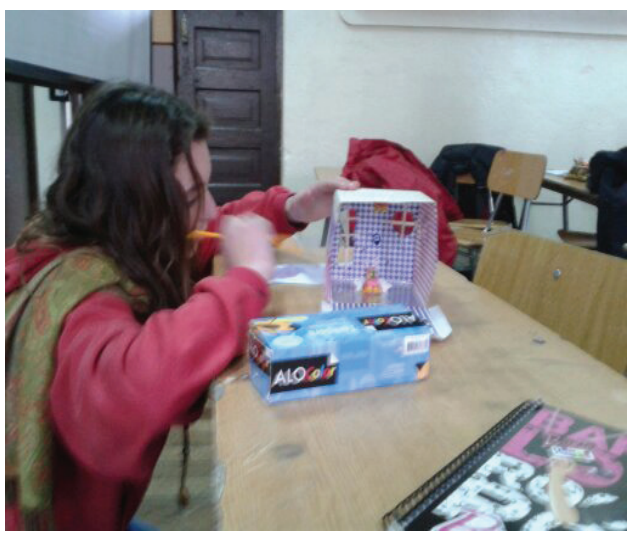

La sesión del 18 de junio del 2014 es un buen reflejo de ese fluir flexible de los y las estudiantes en el proceso de creación:

Mientras la profesora busca un video en internet para ejemplificar el vínculo entre el cubo, ser y contexto, observo que los estudiantes se paran a conversar. Mario le lanza por el aire a Carlos la cinta adhesiva para reforzar el cubo. De pronto se escucha una música fuerte que llama la atención de los estudiantes, todos miran hacia el telón donde está proyectado un video de una mujer acostada sobre una cama con sábanas blancas. Se trata de una secuencia de imágenes fijas que al movimiento da la sensación que el tiempo trascurre. La mayoría ve atenta el video. Mientras, observo a Valeria, ella cuidadosamente toma el cubo entre sus manos, coge el pincel, mira el cubo, y lo pinta de color celeste pastel. Finaliza el video y los estudiantes comienzan el trabajo manual desde sus puestos cortan los cubos, otras ya lo están pintando. Veo a Francisca al final de la sala que baila junto con Marta una cumbia que suena en toda la sala. Observo a Juan, al final de la sala en una cruz improvisada con dos palos, él se toma de los extremos 
simulando estar crucificado. Constanza, por su parte, vende cup cakes que ella misma hace y que sus compañeros sagradamente le compran. Carolina dibuja una silueta de su mano en una hoja, la remarca cuidadosamente. Suena el timbre. Pedro le dice a Claudio, "la prueba hermano", ¿estudiaste?, ¡no! (se toma el pecho).

El extracto del reporte de observación nos muestra cómo cada estudiante y la docente despliegan su accionar desde motivaciones personales, algunas de ellas más vinculadas con la actividad pedagógica general, pero no necesariamente con la acción que la docente estaba promoviendo, ver el video para ejemplificar el vínculo entre ser, cubo y contexto. Varios estudiantes observan el video, Valeria se concentra en la producción de su cubo. A la vez y a continuación del video, cada uno sigue participando, actuando e interactuando desde sus motivaciones: bailan, venden, dibujan, improvisan performance. Parecen acciones inconexas, sin responder a la lógica desde la que la docente planifica la actividad de la sesión, sin embargo, son parte del conjunto del proceso creativo.

Este extracto ofrece otro aspecto interesante para caracterizar el caso analizado, la relación que construyen docente y estudiantes. Pedro dice a Claudio "la prueba hermano", le llama tal como lo hace la docente cuando los interpela. Nos llama la atención cómo ese gesto verbal de referirse al otro no como estudiante, no desde el apellido ni el nombre, sino como "hermano" los ubica en una relación de cercanía, que podría articular cierto sentido de identificación y de un vínculo de reconocimiento entre estudiantes y estudiantes y docente.

La tensión entre el proceso planificado y el proceso recursivo de creación fue más evidente en la Fase de construcción del cubo (Imagen 3). La profesora había estimado dos clases para su construcción, sin embargo los y las estudiantes tardaron siete sesiones. Es interesante cómo la profesora durante la entrevista en un ejercicio reflexivo da cuenta de la misma tensión que observamos y analizamos:

Lo que pasa es que... siento... que de pronto les entregué mucha información. Siento, no sé si lo hice bien cuando preparaba mis Power Point cuando hablaba del ser, del cubo, del contexto. Traté de orientar la actividad, con antecedentes, digamos concretos, pero no sé, si ellos tan rápido comprendieron mi mensaje. Peroooo sí me quedo conforme, porque los trabajos que he visto... sí... habían avances importantes. Lograron comprender... no sé si los tiempos fueron los adecuados... Los tiempos de acción en aula, en eso creo que flaqueé.

La docente siente que si bien la producción de los estudiantes le permite tener cierta certeza de que estos comprendieron y avanzaron, su autocrítica tiene que ver con la planificación original tensionada con los "tiempos de acción en el aula". La preocupación en relación con el aprendizaje de los y las estudiantes la ubica en la comprensión, primero de las instrucciones y toda la información que dio, luego con el nivel de comprensión que se evidencia en los trabajos. Las creaciones le indican que hay avances importantes, diremos nosotras, que hay comprensión en acción material.

\subsection{PRODUCTO ARTÍSTICO. MANIFESTACIÓN DEL APRENDIZAJE}

Al finalizar el proceso de creación se apreció una variedad de formas expresivas y compositivas en los productos intermedios y en los 10 nanometrajes. Los cubos tuvieron diversos protagonismos en la producción final. En una creación dos estudiantes hombres conjugaron el cubo y el ser como un solo personaje. En otra creación dos estudiantes mujeres decidieron incorporar el cubo solo simbólicamente representando dos sentidos que se enfrentaban, oscuridad y luz, y que el personaje principal encarna en el nanometraje 
Estudios Pedagógicos XLI, N Especial: 231-251, 2015

APRENDIZAJE Y PRODUCCIÓN MEDIÁTICA DIGITAL EN LA ESCUELA: UN ABORDAJE ETNOGRÁFICO DEL

APRENDIZAJE COMO PRÁCTICA CULTURAL EN ARTES VISUALES

mediante acciones violentas o pacíficas. En la creación de Alejandra y Marcela, Flor de Vida (Figura 2), el cubo convertido en casa se transforma en un personaje que acompaña al protagonista en distintas etapas de su vida.

Figura 2. Storyboard del Nanometraje Flor de Vida
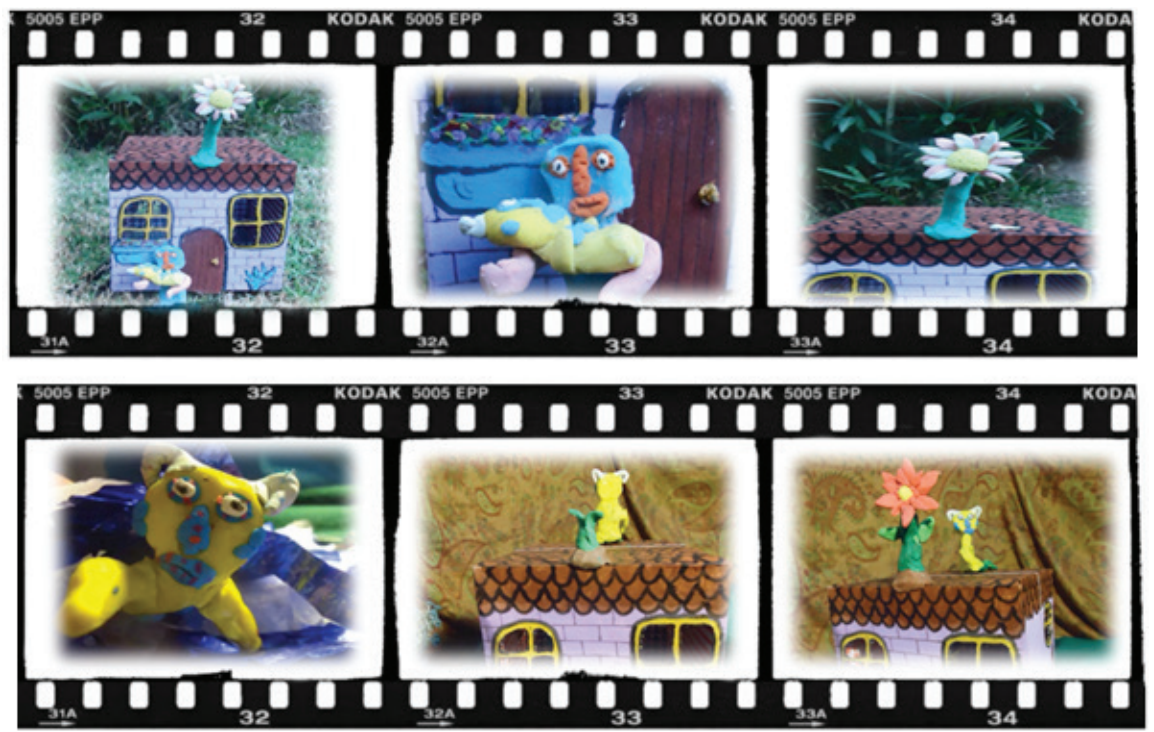

Las creadoras de Flor de vida señalaron en la entrevista que se trata de "una metáfora. Era una flor que al pasar el tiempo se le iban cayendo los pétalos y cada pétalo iba representando como una etapa o un ciclo de la vida". El contexto de este nanometraje es un espacio mágico y fantasioso. Los personajes son seres sin definición humana ni animal. El uso del stop motion, como técnica de producción para dar movimiento a las figuras de plasticina, fue motivación y decisión de las estudiantes. Es interesante, pues tanto la música melódica que acompaña el nanometraje, como la técnica del stop motion, hacen guiños al cine de animación difundido en producciones de Pixar por ejemplo.

Como veremos, las creadoras valoran su proceso y producción por los aprendizajes nuevos en el plano técnico de la expresión. Lo nuevo en esta experiencia fue el uso del video.

\subsection{EL CIERRE DE LA ACTIVIDAD Y LA REFLEXIÓN SOBRE LA CREACIÓN Y EL APRENDIZAJE}

La actividad culmina con la exhibición de los nanometrajes y la retroalimentación de docente y estudiantes. Esto duró cuatro sesiones. Con un computador portátil y un proyector que apuntaba hacia el fondo de la sala, los y las estudiantes giraban sus sillas y sentados se disponían respetuosamente a observar los trabajos de sus compañeros. Cada dupla reproducía su trabajo y explicaba el tema o historia. La Imagen 4 muestra una fotografía de esas sesiones. Este cierre permite el desarrollo del último aprendizaje esperado que la docente declara en su planificación: manifestar juicios críticos sobre las propias creaciones y sus pares y obras. 
Imagen 4. Últimas sesiones durante la exhibición de los nanometrajes

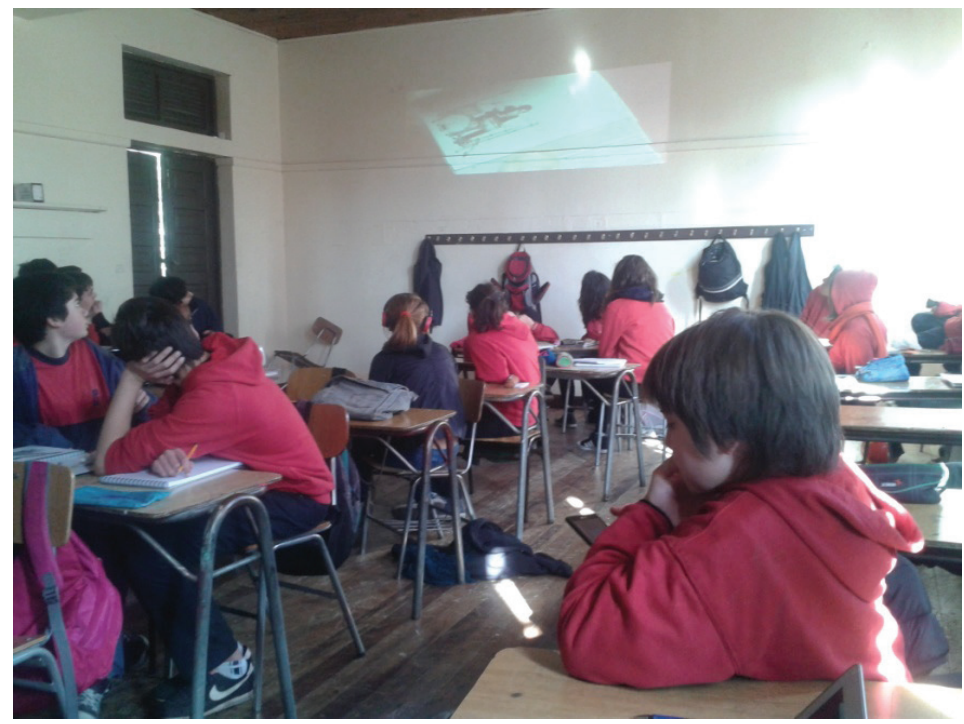

Esta actividad de poner en común los trabajos para la retroalimentación de profesores y estudiantes es parte del cotidiano de las prácticas pedagógicas en este establecimiento, no solo en Artes Visuales. En estas situaciones pudimos observar el despliegue de las experiencias adolescentes para dar y recibir críticas a modo de retroalimentación de los trabajos realizados.

En esta instancia de cierre de la actividad Nanometraje, en la última sesión la docente genera un ambiente especial para evaluar la actividad y dialogar sobre los aprendizajes. A continuación parte del reporte de esa situación.

La profesora reúne a los estudiantes en un círculo. Yo me incorporo al grupo y me siento en una silla expectante a las reflexiones del proceso y, en especial, sobre el aprendizaje, ya que llevo un tiempo cuestionándome si la observación por sí sola contribuye a la comprensión del aprendizaje. La actividad se dilató por meses y en muchas ocasiones tenía la sensación de que los estudiantes no avanzaban en sus trabajos. Sin embargo, a pesar de mis prejuicios, los estudiantes, en un tiempo distinto a lo planeado por la profesora, llegan con sus trabajos, realizados varios fuera del horario de Artes Visuales. En este contexto, en la plenaria la profesora me da la palabra, entonces aprovecho de preguntar sobre qué aprendieron en el proceso del nanometraje, la respuesta de uno de ellos, similar a la de varios: "En realidad lo que más aprendimos fueron cosas técnicas porque fue una distinta manera de armar la creatividad. O sea salir del lápiz o de la hoja (...)".

Tal hallazgo me desestructuró, no esperaba que reconocieran el editar como el único aprendizaje. Luego de unas semanas recordé mi tesis de pregrado, allí una de las principales conclusiones fue que las experiencias de escolarización influyen en los significados de los estudiantes sobre qué es aprender.

Es interesante como las comprensiones diversas sobre la actividad, el proceso y los aprendizajes desplegados dan cuenta no solo de intencionalidades distintas sino que también de 
Estudios Pedagógicos XLI, Nº Especial: 231-251, 2015

APRENDIZAJE Y PRODUCCIÓN MEDIÁTICA DIGITAL EN LA ESCUELA: UN ABORDAJE ETNOGRÁFICO DEL

APRENDIZAJE COMO PRÁCTICA CULTURAL EN ARTES VISUALES

significados e interpretaciones múltiples. Y en este proceso comprensivo de indagar el aprendizaje y la producción mediática (que en este caso asume un acento fundamental en tanto creación), todos y todas participamos, estudiantes, docentes y nosotras como investigadoras.

Ahora bien, la comprensión, como hemos dicho, no es un proceso finito ni clausurado en el momento que ocurre, por lo tanto, tal como dice la observadora en su reporte, la observación es parcial para configurar el aprendizaje. Otros elementos de significación respecto del aprendizaje y la creación mediática aparecen en las entrevistas.

Concluido el proceso, en la entrevista con Alejandra y Marcela se evidencia que el aprendizaje siempre implica algo nuevo, y en este sentido, lo nuevo experimentado fue el uso del audiovisual y todas las herramientas asociadas. Esto porque la experiencia de crear y expresar artísticamente es algo que está dado, que ya es parte del cotidiano en esta clase, con esta profesora y en esta escuela.

Igual la creatividad ya estaba por todo el trabajo anterior, por todos los años con la profesora, pero en realidad, yo creo que lo que más aprendimos fueron cosas técnicas, porque fue una distinta manera de plasmar la creatividad. O sea salir del lápiz o de la hoja, de la pared incluso, salir de eso. Plasmar algo que nunca habíamos hecho antes que era el video (Entrevista de cierre, Marcela y Alejandra).

En otra entrevista, los estudiantes también reconocen y valoran las posibilidades creativas y expresivas que promueven estas actividades, lo que visualizan como algo particular de este contexto donde la docente es pieza clave, pero también el colegio.

Estudiante mujer A: sí, porque igual la profe es como atípica acá dentro de los profes y nos da un espacio mucho más amplio.

Estudiante mujer B: y en comparación con otros colegios. Igual mi vecina, que es mi amiga, también le hacen hacer cosas muy estrictas en artes o en cosas así y no sé, como que acá te dejan fluir. No sé, como expresar.

Este reconocimiento está en sintonía con la intención y motivación de la docente, tanto para la actividad propuesta como para pensar y proyectar el aprendizaje en Artes Visuales. Así se refleja en los siguientes apartados de la entrevista de cierre:

[¿Qué buscaba?] Yo principalmente, al solicitarles un ser... reflexión. Utilizar los recursos que ellos portan a diario como el celular. Que se dieran cuenta que eso podría ser un medio, una herramienta para trabajar también.

[¿Qué y cómo se evalúa en esta experiencia?] El producto artístico al igual que el arte conceptual se vuelve menos importante en términos de resultado apreciable y a evaluar que la idea o impulso emocional orientado artísticamente que permite la expresión fundamental de una intención del ser en colectivo para el colectivo, entonces la idea o conformación emocional en diálogo humano va más allá que un simple resultado físico, evaluable por resultados medibles. La experiencia es la posibilidad de expresión fundamental creativa.

En esta última reflexión se aprecia no solo el valor artístico expresivo que la docente ve en la experiencia de creación en general, sino también su sentido de trascendencia hacia la configuración del ser en colectivo y para el colectivo. Podemos visualizar su posición pedagógica y política respecto de su labor en la enseñanza y el aprendizaje de las Artes 
Visuales, donde la evaluación no debiera estar en el producto físico, medible, si no en la experiencia que orienta artísticamente el impulso emocional para la creación.

\section{DISCUSIÓN Y CONCLUSIONES}

El aprendizaje y la producción mediática como prácticas culturales suponen una serie de elementos que en su articulación configuran procesos y resultados. El caso analizado establece un primer elemento significativo: la producción como acto creativo y artístico. En este caso, la actividad que desarrolla la docente se transforma en una innovación en su práctica pedagógica, ella asume el desafío de acercar las prácticas mediáticas de los adolescentes, incluyendo en la creación lo audiovisual y el celular como recurso tecnológico cotidiano. Tal como señalan Gíraldez y Pimentel (2011), esto en nuestro país se transforma en un desafío e innovación docente por cuanto el currículo de Artes Visuales releva el conocimiento teórico y la expresión plástica sobre otras formas expresivas. Pareciera que la práctica pedagógica asociada a las artes permite una participación menos rígida y encasillada de docente y estudiantes por medio de rutinas que consideran la exploración y la manifestación de intencionalidades diversas.

La tradición histórica de experimentación de la escuela y el vínculo con esta docente son otros componentes de contexto que operan como activo del proceso creativo y del aprendizaje. La participación de los y las estudiantes en la actividad da cuenta de una articulación de orientaciones que provienen tanto de sus experiencias previas en prácticas pedagógicas, donde la autoregulación parece ser un horizonte formativo que está en desarrollo. Los momentos de "no acción pedagógica" como el quedarse mirando el suelo durante largo rato parecen no alterar ni transformarse en problema para la docente. Los resultados observados en los productos permiten a la profesora y etnógrafa tener cierta certeza de que el proceso creativo y de aprendizaje sigue operando más allá del espacio escolar. Otro elemento contextual que aporta es el bagaje de cultura audiovisual de los y las estudiantes, donde el reportorio cinematográfico y las producciones animadas más allá de la oferta televisiva se ofrecen como recursos disponibles para la creación.

Sin embargo, tal como señala Hobart (2010), la práctica del aprendizaje y de la producción mediática son fenómenos complejos y contradictorios. Una primera tensión se dio en la actividad y las orientaciones que estaban detrás. La confrontación de la lógica pedagógica-instruccional y la lógica recursiva y flexible de la creación resulta evidente. La última movilizó a la docente a la apertura, atendiendo a la interacción y propósitos de los estudiantes, sin perder el foco de su intención pedagógica.

Una segunda tensión dice relación con la comprensión que los y las adolescentes tienen sobre aprendizaje, centrado en lo técnico y la novedad, el uso del audiovisual y las herramientas "nuevas" que la docente incluye en su planificación es lo que destacan como aprendizaje. Vemos cómo esto tensiona a la etnógrafa, quien en su búsqueda de comprensión llega a lo mismo planteado por Ruiz (2013): los aprendizajes escolares, en sus sentidos restringidos al espacio instruccional, cuelan los significados adolescentes sobre lo aprendido.

En términos más generales, el caso analizado da cuenta de la complejidad y lo desafiante que resulta para la docencia desarrollar experiencias de producción mediática en el contexto escolar, incluso en este caso particular donde tenemos elementos contextuales históricos y sociales que colaboran. Requiere de la confianza docente y apertura a la incertidumbre 
Estudios Pedagógicos XLI, $\mathrm{N}^{\circ}$ Especial: 231-251, 2015

APRENDIZAJE Y PRODUCCIÓN MEDIÁTICA DIGITAL EN LA ESCUELA: UN ABORDAJE ETNOGRÁFICO DEL

APRENDIZAJE COMO PRÁCTICA CULTURAL EN ARTES VISUALES

respecto de su quehacer y el de sus estudiantes. Demanda revisar las concepciones y formas de evaluar el aprendizaje y de definir el logro de éste; en el caso analizado, la docente lo ubica en la experiencia creativa, por lo tanto en el proceso, que es complejo de observar y conducir de manera uniforme y estandarizada.

Finalmente, quisiéramos destacar la riqueza que aporta la etnografía, como enfoque y método, para dar cuenta de la complejidad de la práctica del aprendizaje en experiencias de producción y creación en el contexto escolar. Creemos que solo un quehacer indagativo que se plantea a sí mismo como situado e integrador de la complejidad que pretende conocer nos acerca a la comprensión del aprendizaje y la acción creativa como práctica cultural.

\section{REFERENCIAS BIBIOGRÁFICAS}

Anderson Levitt, K. (2006). Ethnography. G. Camilli, P. Elmore, \& J. Green (Eds.), Handbook of Complementary Methods in Education Research (pp. 279-295). Washington, DC: American Educational Research Association/Lawrence Erlbaum Associates, Inc.

Arancibia, M., \& Badia, A. (2013). Caracterización y valoración de los usos educativos de las TIC en 10 secuencias didácticas de historia en enseñanza secundaria. Estudios Pedagógicos, XXXIX, Número especial 1, 7-24.

Bergman, M., \& Coxon, A. 2005. The Quality in Qualitative Methods. Forum Qualitative Sozialforschung/Forum: Qualitative SocialResearch, 6(2), (http://nbn-resolving.de/urn:nbn:de:0114fqs0502344). Retrieved from http://www.qualitative-research.net/index.php/fqs/article/view/457/974

Buckingham, D. (2007). Media education goes digital: an introduction. Learning, Media and technology, 32(2), 111-119. doi:10.1080/17439880701343006

Centro de Educación y Tecnología (2014). Informe de resultados SIMCE TIC. $2^{\circ}$ medio (2013). Santiago: Ministerio de Educación.

Centro de Educación y Tecnología; CEPPE; Fundación País Digital (2013). Desarrollo de habilidades digitales para el siglo XXI en Chile. ¿Qué dice el SIMCE TIC? Santiago: Ministerio de Educación.

Cocq, P. (2015). Derechos humanos en el aire: Una propuesta educativa en derechos humanos y habilidades comunicativas orientada a niños y jóvenes vulnerables utilizando la radio. Comunicación y Medios, 0(31), 143-161. doi:10.5354/0719-1529.2015.36043

Couldry, N. (2004). Theorising Media as Practice. Social Semiotics, 14 (2), 115-132.

De Fontcuberta, M.M. (2009). Propuestas para la formación en educación en medios en profesores chilenos. Comunicar, 32, 201-207. doi:10.3916/c32-2009-03-001

Gee, J.P. (2004). Situated language and learning: A critical of traditional schooling. London: Routledge.

Gee, J.P., \& Hayes, E. (2011). Language and Learning in the Digital Age. New York: Routledge.

Giráldez, A., \& Pimentel, L. (2011). Educación artística, cultura y ciudadanía. De la Teoría a la práctica. Madrid, España: Organización de Estados Iberoamericanos para la Educación, la Ciencia y la Cultura (OEI) C/ Bravo Murillo, 38.

Guarinos, V., \& Gordillo, I. (2010). El microrrelato audiovisual como narrativa digital necesaria. Cisti 2010. IX Conferencia Iberoamericana en Sistemas, Cibernética e Informática. Orlando, Florida, USA.

Guber, R. (2004). El Salvaje Metropolitano. Reconstrucción del conocimiento social en el trabajo de campo. Buenos Aires: Paidós.

Guber, R. (2011). La Etnografía. Método, Campo y Reflexividad. Buenos Aires: Siglo XXI Editores.

Hobart, M. 2010. What do we mean by 'media practices'? In B, Bräuchler, \& J. Postill (Eds.), 
Theorising media and practice (pp. 55-75). Oxford: Berg.

Instituto de Informática Educativa Universidad de La Frontera y ADIMARK Gfk. (2013) Censo de Informática Educativa 2012 Resultados Principales. Informe final. Santiago: Ministerio de Educación.

Jenkins, H., Ford, S., \& Green, J. (2013). Spreadable media: Creating value and meaning in a networked culture. New York: New York University Press.

Kennedy, G., Judd, T., Dalgarno, B., \& Waycott, T. (2010). Beyond natives and immigrants: exploring types of net generation students. Journal of Computer Assisted Learning, 26, 332-343. doi: http://dx.doi.org/10.1111/j.1365-2729.2010.00371.x

Lave, J. (1996). La práctica del aprendizaje. S. Chaiklin, \& J. Lave (Comps.). Estudiar las prácticas. Perspectivas sobre actividad y contexto (pp. 15-46). Buenos Aires: Amorrortu Editores.

Lave, J., \& Wenger, E. (1991). Situated Learning: Legitimate Peripheral Participation. New York: Cambridge University Press.

Martín-Barbero, J. (1987). De los medios a las mediaciones. Comunicación, cultura y hegemonía. Barcelona: Anthropos Editorial.

Ministerio de Educación (2009). Objetivos Fundamentales y Contenidos Mínimos Obligatorios de la Educación Básica y Media. Actualización 2009. Santiago: Ministerio de Educación de Chile.

Morduchowicz, R. (2008). La relación de los jóvenes y las pantallas. En R. Morduchowicz (Coord.) Los jóvenes y las pantallas. Nuevas formas de sociabilidad (pp. 47-67). Barcelona: Gedisa.

Prensky, M. (2001). Digital natives, digital inmigrants. On the Horizon, 9(5), 1-6.

Ruiz, P. (2013). Nuevas tecnologías y estudiantes chilenos de secundaria. Aportes a la discusión sobre la existencia de nuevos aprendices. Estudios Pedagógicos, 39(2), 279-298.

Sadik, A. (2008). Digital storytelling: a meaningful technology-integrated approach for engaged student learning. Educational Technology Research and Development, 56(4), 487-506. doi:10.1007/ s11423-008-9091-8.

Säljö, R., \& Wyndhamn, J. (1996). Resolución de problemas cotidianos en un ambiente formal: un estudio empírico de la escuela como contexto para el pensamiento. En S. Chaiklin y J. Lave (Comps.). Estudiar las prácticas. Perspectivas sobre actividad y context (pp. 353-367). Buenos Aires: Amorrortu Editores.

Sánchez, J., Salinas, A., Contreras, D., \& Meyer, E. (2010). Does the new digital generation of learners exist? A Qualitative Study. British Journal of Educational Technology, 42(4), 543-556.

Scheihing, E., Guerra, J., Cárcamo, L., Flores, P., Troncoso, D., \& Aros, C. (2013). La experiencia Kelluwen: Tres años de desarrollo y puesta en práctica de una propuesta de innovación didáctica con uso de TIC. Estudios Pedagógicos, 39(1), 121-141.

Selwyn, N. (2009). The digital native - myth and reality. New Information Perspectives, 61(4), 364-379.

Vigotsky, L. (1978). Pensamiento y lenguaje. Madrid: Paidos.

Wertsch, J. (1991). Voices of the mind: a sociocultural approach to mediated action. Cambridge, M.A.: Harvard University Press. 
